Proceedings

\title{
Health and social care workers' quality of working life and coping while working during the Covid-19 Pandemic: Analysis of positive coping and work-related quality of life as resilience and protective factors impacting on wellbeing ${ }^{\dagger}$
}

Paula McFadden ${ }^{1, *}$, Patricia Gillen ${ }^{2,3}$, John Moriarty ${ }^{4}$, John Mallett ${ }^{5}$, Heike Schroder ${ }^{6}$, Jermaine Ravalier ${ }^{7}$, Jill Manthorpe ${ }^{8}$, Jaclyn Harron ${ }^{9}$, Denise Currie ${ }^{6}$ and Jana Ross ${ }^{1}$

1 Ulster University, Magee campus, Londonderry, BT48 7JL, United Kingdom; p.mcfadden@ulster.ac.uk (P.M.); j.ross@ulster.ac.uk (J.Ro.)

2 Ulster University, Jordanstown campus, Shore Road, Newtownabbey, BT37 0QB, United Kingdom; p.gil$\underline{\text { len@ulster.ac.uk }}$

3 Southern Health and Social Care Trust, 10 Moyallen Road, Gilford, BT63 5JX, United Kingdom; patricia.gillen@southerntrust.hscni.net

4 Queen's University Belfast, 69-71 University Street, Belfast, BT7 1HL, United Kingdom; j.moriarty@qub.ac.uk

5 Ulster University, Coleraine campus, Cromore Road, Coleraine, BT52 1SA, United Kingdom; j.mallett@ulster.ac.uk

6 Queen's University Belfast, 185 Stranmillis Road, Belfast, BT9 5EE, United Kingdom; h.schroder@qub.ac.uk (H.S.); d.currie@qub.ac.uk (D.C.)

7 Bath Spa University, Newton Park, Newton St Loe, Bath, BA2 9BN, United Kingdom; j.ravalier@bathspa.ac.uk

8 King's College London, 22 Kingsway, Holborn, London, WC2B 6LE, United Kingdom; jill.manthorpe@kcl.ac.uk

9 Independent Researcher

* Correspondence: p.mcfadden@ulster.ac.uk

Publisher's Note: MDPI stays neutral with regard to jurisdictional claims in published maps and institutional affiliations.

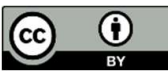

Copyright: (C) 2021 by the authors. Submitted for possible open access publication under the terms and conditions of the Creative Commons Attribution (CC BY) license (http://creativecommons.org/licenses/by/4.0/).

\begin{abstract}
In early 2020, COVID-19 was designated as a pandemic. Despite our experience of pandemics (e.g., SARS), there is limited research about how health and social care staff cope with the challenges of caring for patients while potentially putting their own health at risk. The current study examined the impact of providing health and social care during COVID-19 on nurses, midwives, allied health professionals, social care workers and social workers. An online survey using validated scales and open-ended questions was used to collect data from the UK health and social care staff in May-July 2020. It received 3,290 responses, mostly from social care workers and social workers. A multiple regression analysis showed that individuals using positive coping strategies, particularly active coping, emotional support, relaxation and exercise, had higher wellbeing scores. Lower wellbeing scores were associated with disengagement and substance use as coping strategies. Better quality of working life was associated with active coping, emotional support, work family segmentation and relaxation. Participants using disengagement and family work segmentation had lower quality of working life. Positive coping strategies seem to be playing a significant role in health and social care workers' wellbeing and quality of working life and interventions may be needed to support those who are struggling to cope.
\end{abstract}

Keywords: HSC staff; coping; quality of working life; wellbeing; support; COVID-19

\section{Introduction}

COVID-19 was declared a global pandemic by the World Health Organization in March 2020. Globally, the health and social care systems quickly became overwhelmed 
and were struggling to cope. The impact of the pandemic was, however, felt not only on the organizational level, but also at a more personal level, as studies examining the impacts of the pandemic on individuals' health and wellbeing started emerging [1]. In UK, the health and social care staff are a particularly vulnerable group when it comes to low wellbeing, stress and burnout, perhaps due to the fact that the UK health and social care sector has been under great pressure, understaffed and underfunded, for several years now. The added pressure of the pandemic is likely to have exacerbated the situation, with potentially more health and social care staff feeling stressed, anxious or depressed. There are, however, differences in how people deal with stressful situations, as stress is the interaction between an individual's appraisal of their environment and their appraisal of their own coping resources [2].

The aim of the current study was to examine the impact of providing health and social care in UK during COVID-19 on nurses, midwives, allied health professionals (AHPs), social care workers and social workers. More specifically, the study looked at the relationship between individuals' coping strategies and their levels of wellbeing and work-related quality of life.

\section{Materials and Methods}

\subsection{Data and Participants}

Data was collected through an anonymous questionnaire that was available online between May - July 2020. The study was advertised primarily through regulatory bodies, newsletters and online magazines to nurses, midwives, AHPs, social care workers and social workers. Social media were also used to raise awareness of the study and recruit participants. A total of 3,290 responses were received and pairwise deletion was used in all analyses. Of the responses, 1,897 were from Northern Ireland, 1,062 from England, 185 from Wales and 146 from Scotland. The majority of the sample were social workers $(\mathrm{n}=$ $1,282,38.9 \%)$ and social care workers $(n=1,245,37.8 \%)$, followed by AHPs ( $n=388,11.8 \%)$, nurses $(n=199,6.0 \%)$ and midwives $(n=190,5.5 \%)$. The majority were female $(83.3 \%)$, most belonged to the 40-59 age bracket (63.1\%) and had white ethnic origin (93.3\%). The most common area of work was the community (51.0\%), followed by care homes (13.3\%), hospitals $(12.7 \%)$ and others $(23.1 \%)$. Just over half $(51.3 \%)$ of the sample were married (cohabiting: 20.3\%; single: $21.4 \%$; divorced or separated: $6.9 \%$ ).

\subsection{Measures}

The questionnaire consisted of demographic questions, validated measures of wellbeing, quality of working life and coping, and open-ended qualitative questions.

\subsubsection{Mental wellbeing}

Mental wellbeing was assessed with the Short Warwick Edinburgh Mental Wellbeing Scale (SWEMWBS; [3]), which consists of seven items that are each rated on a five-point Likert scale ranging from $1=$ 'None of the time' to $5=$ 'All of the time'. Participants indicate how often in the last two weeks they felt what is described by each item. Sum scores are created and transformed into metric scores, ranging from 7 to 35). Higher scores indicate better wellbeing.

\subsubsection{Quality of working life}

Quality of working life was assessed using the Work-Related Quality of Life (WRQOL) scale [4], which is a 24-item measure enquiring about one's working conditions. Participants indicate their agreement with the items using a five-point Likert scale ranging from 1 = 'Strongly disagree' to 5 = 'Strongly agree'. Twenty-three items contribute to the final (sum) score, which can range from 23 to 115 and higher scores indicate better quality of working life.

\subsubsection{Coping}


Two measures were used to assess participants' coping strategies. First, participants completed the Brief COPE [5], which consists of 28 items assessing 14 different coping strategies. Using a four-point Likert scale ranging from $1=$ 'I haven't been doing this at all' to $4=$ ' $\mathrm{I}$ 've been doing this a lot', participants indicate how often they do what is described by the items to cope with work-related stress. The scores for each subscale are summed and higher scores indicate that participants use the given coping strategy more often.

Second, participants completed 15 items from a coping scale developed by Clark and colleagues [6]. The items assess five coping strategies (family-work segmentation, workfamily segmentation, working to improve skills/efficiency, recreation/relaxation, exercise) that were selected to supplement the Brief COPE. A six-point Likert scale ranging from 1 $=$ 'Never have done this' to $6=$ 'Almost always do this' was used by participants to indicate how often they do what is described by the items in order to cope with work-related stress. Each one of the five coping strategies is represented by three items. The mean score of these was used in the analysis, with higher scores indicating greater frequency of using the given coping strategy.

\subsubsection{Qualitative questions}

Open-ended questions were used to gain a deeper understanding of the health and social care staff's experiences of working through the pandemic. Relevant to the current study were three questions: 1) 'Can you describe what employer supports have worked well during the COVID-19 pandemic?', 2) 'Can you describe what other support from your employer would have helped you in your day to day job during the COVID-19 pandemic?' and 3) 'If your caring responsibilities have changed during the COVID-19 pandemic, can you say more about this?'.

\subsection{Data analysis}

The data was analysed using descriptive statistics, exploratory factor analysis, multiple regressions and thematic analysis. Descriptive statistics were weighted by participants' occupation (nursing, midwifery, AHP, social care, social work) and country of work (England, Scotland, Wales, Northern Ireland) to account for uneven distribution. The weights were derived from registration numbers in each country. In order to examine the relationships between participants' coping strategies and their mental wellbeing and quality of working life, multiple regressions were conducted with the coping strategies and demographic variables as predictors and mental wellbeing (SWEMWBS) and quality of working life (WRQOL) as outcomes. To reduce the number of predictors, an exploratory factor analysis was conducted with the 28 Brief COPE items. The resulting factors (sum scores of individual items within each factor) were used as predictors, along with the five coping strategies derived from the Clark et al. [6] scale and the following dummy coded demographic variables: age, gender, disability status, ethnic group, country of work, occupational group and the number of sick days in the past 12 months. Qualitative responses were analysed using thematic analysis by two researchers.

\section{Results}

\subsection{Exploratory factor analysis}

Exploratory factor analysis with varimax rotation yielded nine factors, which were interpreted as active coping, behavioural disengagement, religion, acceptance, emotional support, substance use, humour, instrumental support and denial.

\subsection{Wellbeing}

The mean wellbeing score in the sample was 20.95 (SD $=3.78$, range: 7 to 35). The regression model with the SWEMWBS scores as the outcome variable explained 35\% of the variance (adjusted $\mathrm{R}^{2}=.34, \mathrm{~F}(34,2356)=36.5, \mathrm{p}<.001$ ). In terms of the demographic predictors, respondents from Northern Ireland had higher wellbeing scores than those from England $(\beta=0.05, p=.021)$. Participants who were using active coping $(\beta=0.19, p<$ 
$.001)$, emotional support $(\beta=0.15, \mathrm{p}<.001)$, recreation and relaxation $(\beta=0.08, \mathrm{p}<.001)$ and exercise $(\beta=0.07, p<.001)$ as their coping strategies more frequently had significantly higher wellbeing scores. Those who were using primarily behavioural disengagement $(\beta$ $=-0.34, \mathrm{p}<.001)$ and substance use $(\beta=-0.08, \mathrm{p}<.001)$ had significantly lower wellbeing scores.

\subsection{Quality of working life}

The mean WRQOL score was 77.59 (SD = 17.67, range: 23 to 115), indicating average quality of working life for participants in our sample [4]. Based on the normative data [4], $30.4 \%$ of the sample had lower quality of working life, $27.1 \%$ had average quality of working life and $42.5 \%$ had higher quality of working life. The regression model with the WRQOL as the outcome variable explained $26 \%$ of the variance (adjusted $\mathrm{R}^{2}=.25, \mathrm{~F}(33$, $2315)=24.07, p<.001)$. Respondents from Northern Ireland $(\beta=-0.14, p<.001)$ and those from Scotland $(\beta=-0.06, p=.003)$ reported lower WRQOL scores than those from England. Having a disability $(\beta=-0.06, p<.001)$ and the number of sick days in the past 12 months ( $\beta$ range .01 - .05, $\mathrm{p}<.05)$ were also associated with lower WRQOL scores. In terms of participants' coping strategies, those who reported using active coping $(\beta=0.10, \mathrm{p}<.001)$, emotional support $(\beta=0.14, \mathrm{p}<.001)$, recreation and relaxation $(\beta=0.15, \mathrm{p}<.001)$ and work family segmentation $(\beta=0.13, \mathrm{p}<.001)$ had higher WRQOL scores. On the other hand, those who reported using behavioural disengagement $(\beta=-0.31, p<.001)$ and family work segmentation $(\beta=-0.08, \mathrm{p}<.001)$ had lower WRQOL scores.

\subsection{Qualitative responses}

The major themes were consistent across countries and occupational groups and could be summarized under three broad areas: 1) Conditions, 2) Connections, and 3) Communication. These three areas all contained responses from three groups of participants; those with generally positive experiences, those who admitted some work-related challenges and cited areas for improvement, and those who had predominantly negative experiences related to working during the pandemic.

\subsubsection{Changing conditions}

Respondents commented on the stress associated with the rapidly changing conditions brought about by the pandemic. This was primarily related to changes to safety risk, work routines, work intensity and work/home life. In terms of safety, many respondents expressed concerns about contracting the virus and passing it on to their families or service users. This was often related to inadequate provision of personal protective equipment (PPE): "I feel that risk is massively undervalued and under discussed, with regards to our own personal safety. This has been highline more under Covid with regards to PPE... our PPE did not reflect our role and the risk we face" (Social worker, England). Work routines also changed for many respondents due to COVID-19 restrictions, such as those imposed on face-toface service user visits: "Change in the operation of the service with a significant increase in videocalls rather than face to face contacts" (AHP, Northern Ireland). Many respondents appreciated the option to work from home, but for others, this posed significant challenges, particularly in relation to a lack of IT and office equipment. Some had to be redeployed and many felt unprepared for their new roles. Work intensity has also changed during the pandemic, primarily for the frontline staff working in acute COVID-19 response settings. This was compounded by high staff absence levels, as it left those still in work having to do longer hours and at higher intensity: "We have been running with a depleted team due to staff off due to illness, shielding, stress due to the service demand" (Social worker, Northern Ireland). Finally, respondents reported increased levels of stress in their private lives due to increased caring responsibilities (e.g. balancing workload with childcare and home schooling).

\subsubsection{Connections with employers and service users}


Respondents expressed the need for support from employers and managers, such as their recognition for the staff's work, regular contact, checking on staff welfare and formal supervisions. It all came down to the individual line managers, with some respondents being very happy with the support they received: "Having very supportive leaders that I can approach at any time with any problem they have gone that extra mile to enable me to cope in the toughest of times and in very difficult circumstances" (Social care worker, Scotland); and others feeling very dissatisfied or even abandoned: "We haven't heard of our manager barely at all through the pandemic. We have had no direction or advice on our job roles through this all" (Nurse, Wales). A large number of respondents also expressed genuine concerns about their patients/service users: "I am working from home. No face to face contact, so can't physically see if children are safe" (Social worker, Scotland).

\subsubsection{Communication}

Communication was seen as an important factor in staff's experiences during the pandemic. Some were very happy with the effectiveness, regularity and timeliness of the communication from management, often citing IT as the enabling factor. Others, however, felt frustrated, for example, in relation to the changing guidelines and work routines and how these were not communicated by the management or were not tailored to the staff's particular circumstances: "Sometimes the information sent out... was contradictory so that at times I felt supported to work remotely and other times I didn't. This was extremely stressful and worrying (AHP, England)".

\section{Discussion}

This study highlights the importance of coping strategies in mental wellbeing and quality of working life in the health and social care workforce during a pandemic. The regression analyses showed that staff wellbeing and quality of working life vary depending on the types of coping strategies that health and social care workers utilize when faced with stress. Positive coping strategies (e.g., active coping, emotional support, recreation and relaxation) were associated with higher wellbeing and better quality of working life, whereas negative coping strategies (e.g., behavioural disengagement) were associated with lower wellbeing and worse quality of working life. Managers and employers may help to improve these domains of their staff's lives by providing support and guidance, but also more formal training and workshops to equip their workforce with the right tools needed to deal with stressful situations [7]. This may be particularly important as our results show that the level of wellbeing of the health and social care staff in our sample was lower than the UK population average [8], suggesting that health and social care staff may be an at risk group when it comes to lower wellbeing.

The qualitative results support the statistical findings as they show that the staff need the employer and managerial support in order to deal with the demands of stressful circumstances, such as those posed by the COVID-19 pandemic. This is in line with previous research which has shown that supervisor support has a buffering effect against low levels of wellbeing and intention to leave in nurses who have experienced an infection outbreak [9].

\section{Conclusions}

To conclude, the current study highlights the importance of positive coping strategies for health and social care workers' wellbeing and quality of working life during the COVID-19 pandemic. Organisational support in the form of clear and effective communication and connections, particularly during the rapidly changing work conditions, is also important. The findings will be important for managers and employers, not just during the later waves of COVID-19 or other pandemics, but also during business as usual times, as they highlight the needs of the health and social care workforce.

Author Contributions: Conceptualization and methodology, P.M. (PI on the study and lead of social work and social care part of dissemination) and P.G. (CI and lead of health part of the survey); 
CIs and statistical analysis, J.H., J.Mo. and J.M.; Qualitative analysis, P.M., P.G., J.Ra., H.S. and D.C.; Writing - original draft presentation, J.Ro. and P.M.; Writing - review and editing, P.M., P.G., J.Ma. and J.Ro.

Funding: This research was funded by seed funding from the Northern Ireland Social Care Council and the Southern Health and Social Care Trust, and the NIHR Policy Research Programme grant to the NIHR PRU in Health and Social Care Workforce (King's College London).

Acknowledgments: The authors would like to thank Northern Ireland Social Care Council and the Southern Health and Social Care Council, for seed funding for the first survey. Also thanks to Community Care $\left({ }^{\circ}\right.$, Northern Ireland Practice and Education Council for Nursing and Midwifery, Royal College of Nursing, Royal College of Midwifery, Royal College of Occupational Therapists, British Dietetic Association, College of Podiatry and the Northern Ireland Social Care Council for advertising and promoting the study.

Conflicts of Interest: The authors declare no conflict of interest. The funders had no role in the design of the study; in the collection, analyses, or interpretation of data; in the writing of the manuscript, or in the decision to publish the results.

\section{References}

1. Krishnamoorthy, Y.; Nagarajan, R.; Saya, G.K.; Menon, V. Prevalence of psychological morbidities among general population, healthcare workers and COVID-19 patients amidst the COVID-19 pandemic: A systematic review and meta-analysis. Psychiatry Res 2020, 293, 113382. doi: 10.1016/j.psychres.2020.113382

2. Lazarus, R.S.; Folkman, S. Stress, Appraisal and Coping. Springer: New York, 1984.

3. Stewart-Brown, S.; Tennant, A.; Tennant, R.; Platt, S.; Parkinson, J.; Weich, S. Internal construct validity of the Warwick-Edinburgh Mental Well-being Scale (WEMWBS): a Rasch analysis using data from the Scottish Health Education Population Survey. Health Qual Life Outcomes 2009, 7(15), 1-8. doi: 10.1186/1477-7525-7-15

4. Van Laar, D.; Edwards, J.A.; Easton, S. The Work-Related Quality of Life scale for healthcare workers. J Adv Nurs 2007, 60(3), 325-333. doi: 10.1111/j.1365-2648.2007.04409.x

5. Carver, C. S. You want to measure coping but your protocol's too long: Consider the Brief COPE. International Journal of Behavioral Medicine 1997, 4, 92-100. doi: 10.1207/s15327558ijbm0401_6

6. Clark, M.A.; Michel, J.S.; Early, R.J.; Baltes, B.B. Strategies for coping with work stressors and family stressors: Scale development and validation. J Bus Psychol 2014, 29, 617-638. doi: 10.1007/s10869-014-9356-7

7. Chen, H.M.; Huang, M.F.; Yet, Y.C.; Huang, W.H.; Chen, C.S. Effectiveness of coping strategies intervention on caregiver burden among caregivers of elderly patients with dementia. Psychogeriatr 2015, 15, 20-25. doi: 10.1111/psyg.12071

8. Fat, L.N.; Scholes, S.; Boniface, S.; Mindell, J.; Stewart-Brown, S. Evaluating and establishing national norms for mental wellbeing using the short Warwick-Edinburgh mental Well-being Scale (SWEMWBS): findings from the health Survey for England. Qual Life Res 2017, 26(5), 1129-1144. doi: 10.1007/s11136-016-1454-8

9. Jung, H.; Jung, S.Y.; Lee, M.H.; Kim, M.S. Assessing the presence of post-traumatic stress and turnover intention among nurses post-Middle East Respiratory Syndrome outbreak: The importance of supervisor support. Workplace Health Saf 2020, 68(7), 337345. doi: $10.1177 / 2165079919897693$ 